\title{
11D Supergravity as a Gauge Theory for the M-Algebra
}

\author{
Mokhtar Hassaïne, \\ CECS, Valdivia, Chile \\ E-mail: hassaine-at-cecs.cl
}

\section{Ricardo Troncoso}

CECS, Valdivia, Chile

E-mail: ratron-at-cecs.cl

\section{Jorge Zanelli*}

CECS, Valdivia, Chile

E-mail: jz-at-cecs.cl

\begin{abstract}
The eleven-dimensional gravitational action invariant under local Poincaré transformations is given by the dimensional continuation of the Euler class of ten dimensions. Here we show that the supersymmetric extension of this action leads, through the Noether procedure, to a theory with the local symmetry group given by the $M$-algebra. The fields of the theory are the vielbein $e_{\mu}^{a}$, the Lorentz (spin) connection $\omega_{\mu}^{a b}$, one gravitino $\left(\psi_{\mu}\right)$, and two 1-forms, $b_{\mu}^{a b}$ and $b_{\mu}^{a b c d e}$, which transform as antisymmetric Lorentz tensors. These fields are components of a single connection for the $M$-algebra and the supersymmetric Lagrangian can be seen to be a Chern-Simons form. The dynamics has a multiplicity of degenerate vacua without propagating degrees of freedom. The theory is shown to admit solutions of the form $S^{10-d} \times X_{d+1}$, where $X_{d+1}$ is a warped product of $\mathbb{R}$ with a $d$-dimensional spacetime. Among this class, the gravitational effective action describes a propagating graviton only if $d=4$ and the spacetime has positive cosmological constant. The perturbations around this solution reproduce linearized General Relativity around four-dimensional de Sitter spacetime.
\end{abstract}

Fourth International Winter Conference on Mathematical Methods in Physics

09 - 13 August 2004

Centro Brasileiro de Pesquisas Fisicas (CBPF/MCT), Rio de Janeiro, Brazil

\footnotetext{
*Speaker.
} 


\section{Introduction}

Ever since Theodor Kaluza [1] and Oskar Klein [2] conjectured the possibility that fourdimensional spacetime could be a section of a larger manifold where all laws of physics would be unified, theoretical physicists have tried to realize this dream with varying degrees of success. This is such a compelling idea, that many of us believe it must be true at some level, but we have mostly failed to pinpoint exactly how it may work. Among the most trodden paths one finds the attempt to describe the higher-dimensional spacetimes as Einstein did with our own: assuming it faithfully described by the Einstein-Hilbert (EH) action. It turns out that under reasonable assumptions, the Einstein theory is unique -modulo the cosmological constant- only in four dimensions, but is one very particular option among many in higher dimensions. In this lecture, we analyze some consequences of freeing ourselves from the straitjacket of the Einstein-Hilbert action. The result in this case is not, as one may fear, opening a Pandora's box of unlimited possibilities, but a controlled and very restricted set of theories known as Lovelock gravities [3]. These theories extend Einstein's General Relativity, and a very narrow subset of them is known to accept simple supersymmetric extensions ${ }^{1}$.

The standard procedure to carry out the Kaluza-Klein dream is to start with a theory in a sufficiently high dimension whose gravitational sector is given by the EH action. Then, the fourdimensional physical world is expected to arise from compactification of the extra dimensions (see, e.g., [4]), or through some more recent alternatives [5]. This is extremely reasonable and straightforward. The problem is that the fact that the low-energy behavior of the universe is fourdimensional is an assumption rather than a prediction of the theory. This is hardly convincing, specially since the gravitational part of the action should be in charge of determining the properties of the spacetime geometry including, presumably, the explanation for its effective four-dimensional appearance.

A manifestation of this problem is the following paradox: since eleven dimensional Minkowski space is a maximally (super)symmetric state, and the theory is well behaved around it, then, what prompts the space to curl up choosing a particular compactification with less symmetry as the vacuum? And, why not six instead of four dimensions? Ideally, the eleven-dimensional theory should dynamically predict a low energy regime corresponding to a four-dimensional effective theory. In such scenario, a background solution with an effective spacetime dimension greater than four should be expected to be some sort of false vacuum.

The origin of the problem lies in the fact that the dynamics produced by the $\mathrm{EH}$ action is insensitive to dimensionality in the sense that its linearized approximation yields a well behaved wave equation for all dimensions (greater than three). Clearly, the solutions of the equations depend on $D$-in particular, black holes, planetary orbits and life itself would be very different if $D \neq 4-$, but there is no compelling reason, within the theory, why $D$ should be 4.

Here we discuss a theory defined in eleven-dimensional spacetime whose gravitational sector is singled out by requiring the theory to be locally invariant under the Poincare group. With this assumption one can go a long way to specify the entire locally supersymmetric extension and to

\footnotetext{
${ }^{1}$ These are the Chern-Simons (CS) theories of gravity, which exist in dimensions $D=2 n+1$, and possess local off-shell invariance under the $D$-dimensional anti-de Sitter group $S O(D-1,2)$, or its contraction, the Poincaré group, ISO $(D-1,1)$.
} 
study its physical consequences. In particular, after supersymmetrization the theory turns out to be invariant under the symmetry group generated by the the maximal extension of the $\mathcal{N}=1$ superPoincaré algebra in eleven dimensions, commonly known as $M$-algebra. This algebra is spanned by the set of generators $G_{A}=\left\{J_{a b}, P_{a}, Q_{\alpha}, Z_{a b}, Z_{a b c d e}\right\}$, where $J_{a b}$ and $P_{a}$ are the generators of the Poincaré group and $Q_{\alpha}$ is a Majorana spinor supercharge with anticommutator ${ }^{2}$ [11]

$$
\left\{Q_{\alpha}, Q_{\beta}\right\}=\left(C \Gamma^{a}\right)_{\alpha \beta} P_{a}+\left(C \Gamma^{a b}\right)_{\alpha \beta} Z_{a b}+\left(C \Gamma^{a b c d e}\right)_{\alpha \beta} Z_{a b c d e} .
$$

The charge conjugation matrix $C$ is antisymmetric, and the generators $Z_{a b}$ and $Z_{a b c d e}$ are tensors under Lorentz rotations, but otherwise Abelian. This algebra is expected to generate the symmetries of an underlying fundamental theory in eleven dimensions known as M-Theory [8, 9, 10].

Our choice of eleven dimensions may be justified by the wish to explore geometrical and dynamical structures that could be regarded as new "cusps" of the M-theory diagram (see e.g., [6]), in particular because the theory presented here is not equivalent to the standard Cremmer-JuliaScherk supergravity in eleven dimensions [7]. Other dimensions and other symmetry groups -such as the supersymmetric extensions of the AdS group- are also reasonable alternatives.

As shown below, the locally supersymmetric extension of the Poincaré invariant action fixes the field content to include, apart from the graviton $e_{\mu}^{a}$, the spin connection $\omega_{\mu}^{a b}$ and the gravitino $\psi_{\mu}$, two one-form bosonic fields $b_{\mu}^{a b}, b_{\mu}^{a b c d e}$, which are rank two and five antisymmetric tensors under the Lorentz group, respectively. The local supersymmetry algebra closes off-shell, without need for auxiliary fields.

As will be shown, the supersymmetric Lagrangian can be explicitly written as a Chern-Simons form. It is known that for Chern-Simons theories bosonic and fermionic degrees of freedom do not necessarily match, since the dynamical fields are assumed to belong to a connection instead of a multiplet for the supergroup [12] and, as it occurs in three dimensions, there exists an alternative to the introduction of auxiliary fields (see e. g. [13]).

\section{Gravitational sector}

General Relativity in dimensions higher than four is generalized by the so-called Lovelock theories of gravity. These theories respect the assumptions of general covariance, second order field equations for the metric, and they include the Einstein-Hilbert lagrangian as a particular case [3]. The Lovelock Lagrangians are linear combinations of the dimensional continuations of the Euler densities from all lower dimensions [14], namely,

$$
L=\sum_{p=0}^{\left[\frac{D-1}{2}\right]} \alpha_{p} L_{p}
$$

with

$$
L_{p}=\varepsilon_{a_{1} \cdots a_{D}} R^{a_{1} a_{2}} \cdots R^{a_{1 p-1} a_{2 p}} \wedge e^{a_{2 p+1}} \cdots e^{a_{D}},
$$

where $R_{b}^{a}=d \omega_{b}^{a}+\omega_{c}^{a} \omega_{b}^{c}$ is the curvature two-form (wedge products between forms is understood throughout), and $\alpha_{p}$ are arbitrary coefficients. In the series (2.1), $L_{0}$ corresponds to the

\footnotetext{
${ }^{2}$ Note that, contrary to the case in standard supergravity, the generators of diffeomorphisms $\left(\mathcal{H}_{\mu}\right)$ are absent from the right hand side of (1.1).
} 
cosmological constant, $L_{1}$ gives the Einstein-Hilbert Lagrangian and $L_{2}$ is the Gauss-Bonnet term. The Lovelock Lagrangians are by construction invariant under diffeomorphisms and local Lorentz transformations, but in odd dimensions there are special choices of the coefficients $\alpha_{p}$ for which the Lovelock Lagrangians acquire a larger local symmetry: Poincaré or (anti-)de Sitter, as in the next table.

\begin{tabular}{|l|ll|}
\hline$\alpha_{p}$ & Local symmetry $(D=2 n+1)$ \\
\hline Arbitrary & Lorentz & $S O(D-1,1)$, also for even $D]$ \\
$\delta_{p}^{n}$ & Poincaré & $I S O(D-1,1)$ \\
$\frac{l^{2 p-D}}{D-2 p}\left(\begin{array}{l}n \\
p\end{array}\right)$ & anti-de Sitter & $S O(D-1,2)$ \\
$(-1)^{p} \frac{l^{2 p-D}}{D-2 p}\left(\begin{array}{l}n \\
p\end{array}\right)$ & de Sitter & $S O(D, 1)$ \\
\hline
\end{tabular}

Since the action can be expressed in terms of differential forms without using the Hodge dual, these theories cannot yield higher order field equations: In the first order formalism (analogous to Palatini's) the field equations can only involve the first exterior derivatives of the dynamical fields (see, e.g., [15]). Then, if the vanishing torsion condition is imposed, the field equations become at most second order. In the vanishing torsion sector, the theory has the same degrees of freedom as General Relativity [16]. If the torsion constraint is not imposed, the field equations remain first order, even if the theory were coupled to other $p$-form fields without involving the Hodge. In fact, in this way it is impossible to generate higher derivative terms in this theory.

An action with local Poincaré symmetry must be, in particular, invariant under local translations. Since the only field with the right tensor structure to transform as a connection under translations is the vielbein, an infinitesimal local translation must act on the fields as

$$
\delta e^{a}=D \lambda^{a}=d \lambda^{a}+\omega_{b}^{a} \lambda^{b}, \quad \delta \omega^{a b}=0 .
$$

The unique Lovelock lagrangian invariant under local translations is [15, 17],

$$
I_{G}[e, \omega]=\int_{M_{11}} \varepsilon_{a_{1} \cdots a_{11}} R^{a_{1} a_{2}} \cdots R^{a_{9} a_{10}} e^{a_{11}},
$$

which corresponds to choosing the coefficients $\alpha_{p}=\delta_{p}^{n}$.

For the reason given above, we take $I_{G}$ as the gravitational sector of our theory rather than the Einstein-Hilbert action, which is not invariant under $(2.3)^{3}$. The Lagrangian in (2.4) is the tendimensional Euler density continued to eleven dimensions and contains the degrees of freedom of eleven dimensional gravity [16].

A local Poincaré transformation acting on the dynamical fields is a gauge transformation $\delta_{\lambda} A=$ $d \lambda+[A, \lambda]$, with parameter $\lambda=\lambda^{a} P_{a}+\frac{1}{2} \lambda^{a b} J_{a b}$, provided $e^{a}$ and $\omega^{a b}$ are the components of a single connection for the Poincaré group, $A=e^{a} P_{a}+\frac{1}{2} \omega^{a b} J_{a b}$. This observation will be the guiding principle for the construction of a locally supersymmetric extension of $I_{G}$.

\footnotetext{
${ }^{3}$ Under the transformation (2.3), the Einstein-Hilbert action $I_{E H}=\int \varepsilon_{a_{1} \cdots a_{11}} R^{a_{1} a_{2}} e^{a_{3}} \cdots e^{a_{11}}$ changes by a term proportional to $\int \varepsilon_{a_{1} \cdots a_{11}} R^{a_{1} a_{2}} T^{a_{3}} e^{a_{4}} \cdots e^{a_{10}} \lambda^{a_{11}}$, which vanishes only if the torsion equation $T^{a}=D e^{a}=0$ is used. However, this last condition is incompatible with the transformations because $\omega^{a b}$ would be a function of $e^{a}$ and hence, $\delta \omega^{a b}$ would not vanish. See [18]
} 


\section{Supersymmetric extension}

A natural way to construct the locally supersymmetric extension of (2.4) without breaking local Poincaré invariance is by demanding that the new fields required by supersymmetry enter on the same footing as the original ones. In other words, all dynamical fields will be assumed to belong to a single connection for a supersymmetric extension of the Poincare algebra. The simplest option would be to consider the $\mathcal{N}=1$ super Poincaré algebra without central extensions. However, this possibility must be ruled out. Indeed, in this case, the connection would be extended by the addition of a gravitino as $A \rightarrow A+\psi Q$, and the gauge generator would change as $\lambda \rightarrow \lambda+\varepsilon Q$, where $\varepsilon$ is a zero-form Majorana spinor. This fixes the supersymmetric transformations to be

$$
\delta_{\varepsilon} e^{a}=\bar{\varepsilon} \Gamma^{a} \psi, \quad \delta_{\varepsilon} \psi=D \varepsilon, \quad \delta_{\varepsilon} \omega^{a b}=0 .
$$

Then, the variation of (2.4) under supersymmetry is canceled by a kinetic term for the gravitino of the form

$$
I_{\psi}=-\frac{1}{3} \int_{M_{11}} R_{a b c} \bar{\psi} \Gamma^{a b c} D \psi
$$

where $R_{a b c}:=\varepsilon_{a b c a_{1} \cdots a_{8}} R^{a_{1} a_{2}} \cdots R^{a_{7} a_{8}}$. The variation of $I_{\psi}$ produces, in turn, an extra piece which cannot be canceled by a local Lagrangian for $e^{a}, \omega^{a b}$, and $\psi$, and hence the super Poincaré algebra is not rich enough to ensure the off-shell supersymmetry of the action.

On the other hand, following the Noether procedure, it can be seen that supersymmetry may be achieved if additional bosonic fields are introduced. These fields can only be a second-rank or a fifth-rank tensor one-forms $b^{a b}$, and $b^{a b c d e}$, which transform as

$$
\delta_{\varepsilon} b^{a b}=\bar{\varepsilon} \Gamma^{a b} \psi, \quad \delta_{\varepsilon} b^{a b c d e}=\bar{\varepsilon} \Gamma^{a b c d e} \psi,
$$

respectively.

Assuming that the dynamical fields belong to a single connection for a supersymmetric extension of the Poincare group, the only option that brings in these extra bosonic fields is to consider the M-algebra (1.1). Additionally, this also prescribes their supersymmetry transformations in the expected form (3.3). This means that the field content is given by the components of a single fundamental field, the M-algebra connection,

$$
A=\frac{1}{2} \omega^{a b} J_{a b}+e^{a} P_{a}+\psi^{\alpha} Q_{\alpha}+b^{a b} Z_{a b}+b^{a b c d e} Z_{a b c d e},
$$

and the required local supersymmetry transformations $(3.1,3.3)$ are obtained from a gauge transformation of the M-connection (3.4) with parameter $\lambda=\varepsilon^{\alpha} Q_{\alpha}$.

Thus, the supersymmetric extension of (2.4) is found to be

$$
\begin{aligned}
I_{\alpha}= & I_{G}+I_{\psi}-\frac{\alpha}{6} \int_{M_{11}} R_{a b c} R_{d e} b^{a b c d e} \\
& +16(1-\alpha) \int_{M_{11}}\left[R^{2} R_{a b}-6\left(R^{3}\right)_{a b}\right] R_{c d}\left(\bar{\psi} \Gamma^{a b c d} D \psi-6 R^{[a b} b^{c d]}\right),
\end{aligned}
$$

where $R^{2}:=R^{a b} R_{b a}$ and $\left(R^{3}\right)^{a b}:=R^{a c} R_{c d} R^{d b}$. Here $\alpha$ is a dimensionless constant whose meaning will be discussed below. 
This action is invariant under $(2.3,3.1,3.3)$, local Lorentz rotations, and also under the local Abelian transformations

$$
\delta b^{a b}=D \theta^{a b}, \delta b^{a b c d e}=D \theta^{a b c d e} .
$$

Invariance under general coordinate transformations is guaranteed by the use of exterior forms. It is simple to see that the local invariances of the action, including Poincare transformations, supersymmetry and the Abelian transformations (3.6), are obtained by a gauge transformation of the M-connection (3.4) with parameter $\lambda=\lambda^{a} P_{a}+\frac{1}{2} \lambda^{a b} J_{a b}+\theta^{a b} Z_{a b}+\theta^{a b c d e} Z_{a b c d e}+\varepsilon^{\alpha} Q_{\alpha}$. As a consequence, the off-shell invariance of the action under the supersymmetry algebra is ensured by construction without invoking field equations or requiring auxiliary fields.

\subsection{Manifest M-covariance}

The action (3.5) describes a gauge theory for the M-algebra with fiber bundle structure, which can be seen explicitly by writing the Lagrangian as a Chern-Simons form [19] for the M-connection (3.4). Indeed, the Lagrangian satisfies $d L=\left\langle F^{6}\right\rangle$, where the curvature $F=d A+A^{2}$ is given by

$$
F=\frac{1}{2} R^{a b} J_{a b}+\tilde{T}^{a} P_{a}+D \psi^{\alpha} Q_{\alpha}+\tilde{F}^{[2]} Z_{[2]}+\tilde{F}^{[5]} Z_{[5]},
$$

with $\tilde{T}^{a}=D e^{a}-(1 / 2) \bar{\psi} \Gamma^{a} \psi$ and $\tilde{F}^{[k]}=D b^{[k]}-(1 / 2) \bar{\psi} \Gamma^{[k]} \psi$ for $k=2$ and 5. The bracket $\langle\ldots\rangle$ stands for a multilinear form of the M-algebra generators $G_{A}$ whose explicit expression is far from obvious. In the case at hand, it can be shown that the only nonvanishing components of the bracket are given by

$$
\begin{aligned}
& \left\langle J_{a_{1} a_{2}}, \cdots, J_{a_{9} a_{10}}, P_{a_{11}}\right\rangle=\frac{16}{3} \varepsilon_{a_{1} \cdots a_{11}}, \\
& \left\langle J_{a_{1} a_{2}}, \cdots, J_{a_{9} a_{10}}, Z_{a b c d e}\right\rangle=-\alpha \frac{4}{9} \varepsilon_{a_{1} \cdots a_{8} a b c} \eta_{\left[a_{9} a_{10}\right][d e]}, \\
& \left\langle J_{a_{1} a_{2}}, J_{a_{3} a_{4}}, J_{a_{5} a_{6}}, J^{a_{7} a_{8}}, J^{a_{9} a_{10}}, Z^{a b}\right\rangle=(1-\alpha) \frac{16}{3}\left[\delta_{a_{1} \cdots a_{10} a b}^{a_{7} \cdots a_{6}}-\delta_{a_{1} \cdots a_{4}}^{a_{9} a_{10} a_{b}} \delta_{a_{5} a_{6}}^{a_{7} a_{8}}\right] \\
& \left\langle Q, J_{a_{1} a_{2}}, J^{a_{3} a_{4}}, J^{a_{5} a_{6}}, J^{a_{7} a_{8}}, Q\right\rangle=\frac{32}{15}\left[C \Gamma_{a_{1} a_{2}}^{a_{3} \cdots a_{8}+}\right. \\
& \left.\qquad(1-\alpha)\left(3 \delta_{a_{1} a_{2} a b}^{a_{3} \cdots a_{6}} C \Gamma^{a_{7} a_{8} a b}+2 C \Gamma^{a_{3} \cdots a_{6}} \delta_{a_{1} a_{2}}^{a_{7} a_{8}}\right)\right],
\end{aligned}
$$

where (anti-)symmetrization under permutations of each pair of generators is understood when all the indices are lowered ${ }^{4}$. The explicit form of this bracket allows writing the field equations in a manifestly covariant form as

$$
\left\langle F^{5} G_{A}\right\rangle=0
$$

In addition, if the eleven-dimensional spacetime is the boundary of a twelve-dimensional manifold, $\partial \Omega_{12}=M_{11}$, the action (3.5) can also be written as $I=\int_{\Omega_{12}}\left\langle F^{6}\right\rangle$, which describes a topological theory in twelve dimensions. In spite of its topological origin, the eleven-dimensional action does possess propagating degrees of freedom and hence it should not be thought of as a topological field theory.

\footnotetext{
${ }^{4}$ In general, without an explicit representation of the algebra, it is a highly nontrivial exercise to determine the existence and the form of an invariant tensor of arbitrary rank.
} 


\section{Gravitons and four-dimensional spacetime}

We now turn to the problem of identifying one true vacuum of the theory. Obviously, a configuration with a locally flat connection, $F=0$, solves the field equations (3.7) and would be a natural candidate for vacuum in a standard field theory. Moreover, such state is invariant under all gauge transformations and is, therefore, maximally supersymmetric under (3.1) and (3.3) which makes it likely to be a stable (BPS) configuration.

The identification of this solution with a vacuum state is more compelling in view of the fact that it carries no charges of any kind and invariant under spacetime transformations and supersymmetry. Matter-free eleven-dimensional Minkowski spacetime is an example of this. However, no local degrees of freedom can propagate on such background: all perturbations around it are zero modes.

In a matter-free configuration, $\psi=0, b^{[2]}=0, b^{[5]}=0$, Eq. (3.7) is a set of polynomial equations of fifth degree in the curvature two-forms. In particular, the equations obtained varying with respect to the vielbein and the spin connection are

$$
\begin{gathered}
\varepsilon_{A A_{1} \cdots A_{10}} R^{A_{1} A_{2}} \cdots R^{A_{9} A_{10}}=0 \\
\varepsilon_{A B A_{1} \cdots A_{9}} R^{A_{1} A_{2}} \cdots R^{A_{7} A_{8}} T^{A_{9}}=0 .
\end{gathered}
$$

Thus, in order to have a propagating connection, the spatial components $R_{i j}^{A B}$ cannot be small. Alternatively, a deviation that propagates around a flat background $R^{A B}=0$ cannot be infinitesimal and is therefore non-perturbative. Moreover, since the derivatives of the field cannot be small either, the deviations are necessarily non-local. This feature is not altered by the remaining equations. Alternatively, in order to have well-defined linearized perturbations, a background solution must be a simple zero of one of the set of equations. In particular, this requires the curvature to be nonvanishing on a submanifold of a large enough dimension.

\subsection{Nontrivial vacuum geometry}

Let us consider a torsionless spacetime with a product geometry of the form $X_{d+1} \times S^{10-d}$, where $X_{d+1}$ is a domain wall whose worldsheet is a $d$-dimensional spacetime $M_{d}$. The line element is given by

$$
d s^{2}=e^{-2 \xi|z|}\left(d z^{2}+\tilde{g}_{\mu \nu}^{(d)}(x) d x^{u} d x^{v}\right)+\gamma_{m n}^{(10-d)}(y) d y^{m} d y^{n},
$$

where $\tilde{g}_{\mu \nu}^{(d)}$ stands for the worldsheet metric with $\mu, v=0, \ldots, d-1 ; \gamma_{m n}^{(10-d)}$ is the metric of $S^{10-d}$ of radius $r_{0}$ and $\xi$ is a constant. This Ansatz solves (4.2) identically, while (4.1) takes the form

$$
\varepsilon_{a a_{1} b_{1} \cdots a_{k} b_{k} m_{1} \cdots m_{s}}\left[\tilde{R}^{a_{1} b_{1}}-\xi^{2} \tilde{e}^{a_{1}} \tilde{e}^{b_{1}}\right] \cdots\left[\tilde{R}^{a_{k} b_{k} 1}-\xi^{2} \tilde{e}^{a_{k}} \tilde{e}^{b_{k}}\right] e^{m_{1}} \cdots e^{m_{s}}=0
$$

where $\tilde{e}^{a}$ and $\tilde{R}^{a b}$ stand for the vielbein and the Riemann curvature of the worldsheet, respectively, and $k=\left[\frac{d-1}{2}\right]$, with $2 k+s=10$.

A solution for equations (4.4) is found if the worldsheet has constant curvature,

$$
\tilde{R}^{a b}-\xi^{2} \tilde{e}^{a} \wedge \tilde{e}^{b}=0 .
$$


This means that $M_{d}$ can be either locally de Sitter spacetime of radius $\xi^{-1}$, or locally Minkowski, if $\xi=0$. The requirement that the curvature of (4.3) be a simple zero of the field equations, implies that $k$ must be one, and therefore $d$ can only be equal to three or four. The case $d=3$ is readily discarded as it possesses no perturbations, as in standard three-dimensional gravity. For $d=4$, the only relevant equation for the perturbations is the one that arises from the variation with respect to $\tilde{e}^{i}$, the perturbations of the spacetime metric satisfy

$$
\xi \delta(z) \varepsilon_{i j k l} \delta\left(\tilde{R}^{j k}-\xi^{2} \tilde{e}^{j} \tilde{e}^{k}\right) \tilde{e}^{l}=0 .
$$

Since for $\xi=0$ the perturbation equations (4.5) become empty, Minkowski spacetime must be ruled out. In sum, the existence of the propagator requires $d=4$, and the four-dimensional cosmological constant to be strictly positive, $\Lambda_{4}=3 \xi^{2}$.

Note that Eq. (4.5) has support only on the $z=0$ plane. Perturbations along the worldsheet, $\delta \tilde{g}_{\mu v}=h_{\mu v}(x)$ reproduce the linearized Einstein equations in four-dimensional de Sitter spacetime. The modes that depend on the coordinates transverse to the worldsheet fall into two classes: those of the form $\delta \tilde{g}_{\mu v}=h_{\mu v}(x, y)$ are massive Kaluza-Klein modes with a discrete spectrum, while $\delta \tilde{g}_{\mu v}=h_{\mu v}(x, z)$ correspond to Randall-Sundrum-like massive modes whose spectrum is continuous and has a mass gap. The perturbations of the remaining metric components are zero modes, which is related to the fact that the equations are not deterministic for the compact space.

\section{Discussion}

A. We have presented a framework in which only a four-dimensional spacetime can sustain propagating gravitons. The mechanism is based on an eleven-dimensional action which is a gauge theory for the M-algebra. Its is shown that the Lagrangian can be written as a Chern-Simons form. The possibility of dynamical dimensional reduction arises because the theory has radically different spectra around backgrounds of different effective spacetime dimensions. Thus, in a family of product spaces of the form $X_{d+1} \times S^{10-d}$, the only option that yields a well defined low energy propagator for the graviton is $d=4$ and $\Lambda_{4}>0$.

It should be stressed that for all gravity theories of the type discussed here, possessing local Poincaré invariance in dimensions $D=2 n+1 \geq 5$, four-dimensional de Sitter spacetime is also uniquely selected by the same mechanism as the background for the low-energy effective theory [20]. This is in sharp contrast with the scenario in a (super)gravity theory based on the EinsteinHilbert Lagrangian for the gravitational sector. In the standard cases, the kinetic term for the metric and, consequently, the propagator is equally well behaved (no degeneracies arise) in all dimensions. In other words, there is nothing in these theories that can produce different dynamical behaviors for different dimensions. This may help to resolve the paradox of compactification mentioned in the introduction: what prompts the stable Minkowski space to curl up, loosing symmetries, and why does it compactify into $4+(D-4)$ and not otherwise?

B. The eleven-dimensional action (3.5) has a free parameter $\alpha$, which reflects the fact that the theory contains two natural limits, corresponding to different subalgebras of (1.1). For $\alpha=0$, the action $I_{0}$ in Eq. (3.5) does not depend on $b^{[5]}$ and corresponds to a gauge theory for the supermembrane algebra, while for $\alpha=1$, the bosonic field $b^{[2]}$ decouples, and $I_{1}$ is a gauge theory for the 
super five-brane algebra as discussed in [21]. It is interesting to note that the linear combination of both limits, $I_{\alpha}=I_{0}+\alpha\left(I_{1}-I_{0}\right)$, is not only invariant under the intersection of both algebras, but under the entire $\mathrm{M}$-algebra. As the term $I_{1}-I_{0}$ does not couple to the vielbein and is invariant under supersymmetry by itself, $\alpha$ is an independent coupling constant. A similar situation occurs in nine dimensions where, in one limit, the theory corresponds to the super five-brane algebra, while for the other it is a gauge theory for the super-Poincaré algebra with a central extension [22].

C. In the presence of negative cosmological constant, eleven-dimensional AdS supergravity [12] can be written as a Chern-Simons theory for $\operatorname{osp}(32 \mid 1)$, which is the supersymmetric extension of $\mathrm{AdS}_{11}$. It is natural to ask whether there is a link between that theory in the vanishing cosmological constant limit, and the one discussed here. Since the M-algebra has 55 bosonic generators more than $\operatorname{ssp}(32 \mid 1)$, these theories cannot be related through a Inönü-Wigner contraction for a generic value of $\alpha$. However, it has been recently pointed out in [23], generalizing the procedure of [24], that it is possible to obtain the M-algebra from an expansion of $\operatorname{ssp}(32 \mid 1)$. In this light, applying this procedure to the eleven-dimensional AdS supergravity theory, it should be expected that the action presented here will be recovered up to some additional terms decoupled from the vielbein, that are supersymmetric by themselves.

Acknowledgments.- We thank J. Edelstein, G. Kofinas, C. Martínez, C. Núñez, R. Portugués and C. Teitelboim for many enlightening discussions and useful comments. This work is partially supported by grants 3020032, 1010450, 1010446, 1010449, 1020629 and 1040921 from FONDECYT. We thank the organizers of Fourth International Winter Conference on Mathematical Methods in Physics for the lively and stimulating conference in Rio. Institutional support to the Centro de Estudios Científicos (CECS) from Empresas CMPC is gratefully acknowledged. CECS is a Millennium Science Institute and is funded in part by grants from Fundación Andes and the Tinker Foundation.

\section{References}

[1] Th. Kaluza, On the Problem of Unity in Physics, Sitzungsber. Preuss. Akad. Wiss. Berlin (Math.Phys.) 1921 (1921) 966.

[2] O. Klein, Quantum Theory and Five-Dimensional Theory of Relativity, Z. Phys. 37 (1926) 895; Surveys High Energ. Phys. 5 (1986) 241.

[3] D. Lovelock, The Einstein Tensor and its Generalizations, J. Math. Phys. 12 (1971) 498.

[4] M. J. Duff, B. E. W. Nilsson and C. N. Pope, Kaluza-Klein Supergravity, Phys. Rept. 130 (1986) 1.

[5] N. Arkani-Hamed, S. Dimopoulos and G. R. Dvali, The Hierarchy Problem and New Dimensions at a Milimeter, Phys. Lett. B429 (1998) 263 [hep-ph/9803315]; L. Randall and R. Sundrum, A Large Mass Hierarchy from a Small Extra Dimension, Phys. Rev. Lett. 83 (1999) 3370 [hep-ph/9905221].

[6] B. de Wit, H. Nicolai and H. Samtleben, Gauged Supergravities in Three Dimensions: A Panoramic Overview, talks given at 27th Johns Hopkins Workshop on Current Problems in Particle Theory: Symmetries and Mysteries of M-Theory, Goteborg, Sweden, 24-26 Aug 2003, hep-th/0403014.

[7] E. Cremmer, B. Julia and J. Scherk, Supergravity Theory in Eleven-Dimensions, Phys. Lett. B76 (1978) 409. 
[8] P. K. Townsend, The Eleven-Dimensional Supermembrane Revisited, Phys. Lett. B350 (1995) 184 [hep-th/9501068].

[9] E. Witten, String Theory Dynamics in Various Dimensions, Nucl. Phys. B443 (1995) 85 [hep-th/9503124].

[10] J. H. Schwarz, The Power of M-Theory, Phys. Lett. B367 (1996) 97 [hep-th/9510086].

[11] P. K. Townsend, P-Brane Democracy, hep-th/9507048.

[12] R. Troncoso and J. Zanelli, New Gauge Supergravity in Seven-Dimensions and Eleven-Dimensions, Phys. Rev. D58 (1998) 101703 [hep-th/9710180]; Gauge Supergravities for All Odd Dimensions, Int. J. Theor. Phys. 38 (1999) 1181 [hep-th/9807029].

[13] P. S. Howe, J. M. Izquierdo, G. Papadopoulos and P. K. Townsend, New Supergravities with Central Charges and Killing Spinros in (2+1)-Dimensions, Nucl. Phys. B467 (1996) 183 [hep-th/9505032].

[14] B. Zumino, Gravity Theories in More than Four-Dimensions, Phys. Rept. 137 (1986) 109.

[15] R. Troncoso and J. Zanelli, Higher Dimensional Gravity, Propagating Torsion and ADS Gauge Invariance, Class. Quant. Grav. 17 (2000) 4451 [hep-th/9907109].

[16] C. Teitelboim and J. Zanelli, Dimensionally Continued Topological Gravitation Theory in Hamiltonian Form, Class. Quant. Grav. 4 (1987) L125.

[17] A. H. Chamseddine, Topological Gravity and Supergravity in Various Dimensions, Nucl. Phys. B346 (1990) 213.

[18] T. Regge, On Broken Symmetries and Gravity, Phys. Rept. 137 (1986) 31.

[19] See, e. g., M. Nakahara, Geometry, Topology and Physics, Adam Hilger, Bristol 1991.

[20] M. Hassaïne, R. Troncoso, and J. Zanelli, Poincaré Invariant Gravity with Local Supersymmetry as a Gauge Theory for the M-Algebra, Phys. Lett. B596(2004) 132 [hep-th/0306258].

[21] M. Bañados, R. Troncoso and J. Zanelli, Higher Dimensional Chern-Simons Supergravity, Phys. Rev. D54 (1996) 2605 [gr-qc/9601003].

[22] M. Hassaïne, R. Olea and R. Troncoso, Supersymmetric Extension of the Nine-Dimensional Continuation of the Euler Density with N=2, Phys. Lett. B599 (2004) 111 [hep-th/0210116].

[23] J. A. de Azcárraga, J. M. Izquierdo, M. Picón and O. Varela, Generating Lie and Gauge Free Differential (Super)Algebras by Expanding Maurer-Cartan Forms and Chern-Simons Supergravity, Nucl. Phys. B662 (2003) 185 [hep-th/0212347].

[24] M. Hatsuda and M. Sakaguchi, Wess-Zumino Term for the ADS Superstring and Generalizes Inonu-Wigner Contraction, Prog. Theor. Phys. 109 (2003) 853 [hep-th/0106114]. 